\title{
DECENTRALIZING OF TRANSPARENCY: USING BLOCKCHAIN TO REDUCE COUNTERFEITING
}

\author{
Eduard Daoud \\ Technical university of Chemnitz, Str. der Nationen 62, 09111 Chemnitz, Germany
}

\begin{abstract}
Whether online or in-store, how can consumer trust and interact with certification marks? The lack of transparency when buying a product with a certificate is one of the shadows when economic coming to growth. According to the report conducted jointly by the Organization for Economic Co-operation and Development (OECD) and the EU Intellectual Property Office (EUIPO) on "Trade in Counterfeit and Pirated Goods: Mapping the Economic Impact", imports of counterfeit and pirated goods are worth nearly half a trillion dollars a year, or around $2.5 \%$ of global imports. In the EU, counterfeit and pirated goods amounted to up to $5 \%$ of imports or as much as EUR 85 billion (USD 116 billion) (OECD/EUIPO, 2016). The Increasing presence of counterfeit and non-compliant (often unsafe) products on the European market need from one side the intervention of Inspection Agencies and from the other side a Decentralizing of Transparency by using Blockchain Technology. So far, approximately 50 alerts published each week on the European Commission's website and just over 2000 alerts published per year about counterfeiting (Directorate-General for Justice and Consumers (European Commission), 2018). In this paper, we describe a decentralized product certificate information that ensures consumer inform themselves about product specification especially the validity information of the certificate. We implement a protocol that turns a Blockchain into an automated access-control. Unlike Bitcoin, transactions in our system are not strictly financial - they are used to carry data, such as storing, querying and sharing product certificate data after that we briefly examine and substantiate short why the Industry and society need a new approach to reduce counterfeiting and improve transparency and what is the difference to the conventional way to access with a central database. Finally, we discuss possible future extensions to Blockchains that could harness them into a well-rounded solution for trusted computing problems in society. One of the main Goals of this research is we went to enable consumer to check the validity of certifications, understand their value. This would be the one of the important steps in the direction to reduce counterfeiting. Like all prior disruptive technologies there will be beneficial and detrimental aspects of Blockchain technologies that will need to be carefully considered prior to development and commercialization of the approach presented in this paper.
\end{abstract}

\section{KEYWORDS}

Blockchain, Counterfeiting, Decentralization, Information Access

\section{INTRODUCTION AND THE CURRENT PROBLEM}

Whether online or in-store, how can consumer trust and interact with certification marks? The lack of transparency when buying a product with a certificate is one of the shadows when economic coming to growth. According to the report conducted jointly by the Organization for Economic Co-operation and Development (OECD) and the EU Intellectual Property Office (EUIPO) on "Trade in Counterfeit and Pirated Goods: Mapping the Economic Impact", imports of counterfeit and pirated goods are worth nearly half a trillion dollars a year, or around $2.5 \%$ of global imports. In the EU, counterfeit and pirated goods amounted to up to $5 \%$ of imports or as much as EUR 85 billion (USD 116 billion) (OECD/EUIPO, 2016). A Rapid Alert System is since its creation in 2003 provide EU Member States a network and communication tools to published Alters about counterfeit products. The system is stabilizing at a regular rate with approximately 50 alerts published each week on the European Commission's website and just over 2000 alerts published per year. (Directorate-General for Justice and Consumers (European Commission), 2018). The number of counterfeits reported products is extremely low in relation to the number of counterfeit products imported into the EU.

Substandard counterfeit products, such as toys and household appliances, can pose significant health and safety threats for consumers. Policy makers are placing emphasis on combating counterfeit and pirated trade. 
This has been paralleled by increased efforts by the Inspection Agencies to raise awareness of this risk (TÜV SÜD AG). However, initiatives to counter counterfeit trade have been hampered by a lack of robust, quantitative information on the magnitude and scope of the problem worldwide. (OECD/EUIPO, 2016, S. 16). Otherwise the end consumer has no options to easily and confidently inspect product certificates, check the validity information of the certificate and interact with the certification marks.

A central database for all certified product could be a way to reach a single point of transparency but such a platform depandes in the first line on the certification bodies and Inspection Agencies which are in competitive relationship with each other, this reduces the willingness to implement such solutions. Much more important is the Data Inaccuracy and how easy to fake certificate to go to the market. TÜV SÜD AG is one of the global player in this area publish on its website the misuse of their quality marks as a list (TÜV SÜD AG, S. Misuse of Quality marks ) more than 350 certificate are affected. The certification bodies and Inspection Agencies need to rethink how they publish and provide their certification in the next section we will briefly examine and substantiate short why the Industry and society need a new approach to reduce counterfeiting and improve transparency.

\section{WHY THE INDUSTRY AND SOCIETY NEED THIS APPROACH TO BE IMPLEMENTED?}

Firstly, a Decentralizing of the certification data will support the work of sustainability standards organizations further information on this point will be elaborated in section 3. Secondly, there is an increasing number of counterfeit and non-compliant (often unsafe) products on the European market which needs to be addressed (OECD/EUIPO, 2016). Only 50 alerts published each week on the European Commission's website and just over 2000 alerts published per year this number is exermly low in realtion to the number of counterfeit and non-compliant (often unsafe) products on the European market. (Directorate-General for Justice and Consumers (European Commission), 2018)

Another reason that the Netherlands Court of Audit conducted an audit on the European system of CE marking and published a report on CE marking on 19 January 2017. The report indicates that dozens of products bearing a CE marking are taken off the market every year because they present a grave risk to the health and safety of consumers. Consumers buying a product with the letters $\mathrm{CE}$ on it cannot assume that the product is safe, healthy or environmentally friendly. On average, 800 products subject to the CE system have to be withdrawn from the European market every year because they are neither safe nor healthy (The Netherlands Court of Audit, 2017).

Besides, customs and market surveillance authorities have limited abilities to identify products with high risks of non-conformity or counterfeiting, $0.3 \%$ of products entering the EU are inspected by market surveillance authorities. Inspections by Member States between 2010 and 2013 revealed non-compliance in $32 \%$ of inspected toys, $47 \%$ of construction products and $58 \%$ of electromagnetic and radio equipment. (Sirpa, Françoise, \& Bart). For normal consumer, there is currently no common platform for verifying authenticity and conformity of products. Limited enforcement resources (customs and market surveillance authorities) also make the problem worse (Directorate-General for Justice and Consumers (European Commission), 2018). Due to this, members of the European Parliament call on Members of the European Commission on the urgent need to address the challenges of market surveillance in Europe (Remeur, 2017).

After we briefly examined and substantiated short why the Industry and society need a new approach to reduce counterfeiting and improve transparency. In the next section, the Blockchain-based approach would be explained afterwards a comparison between centralized-based and decentralized-based approach underpinned with a proof of concept finally the challenges, Conclusion and Future works.

\section{BLOCKCHAIN-BASED APPROACH}

The new approach goal is reducing counterfeiting and improve transparency by better traceability and Immutability. As Blockchain technology becomes firstly embedded in the finance industries (Nakamoto, 2008) and the potential for additional Blockchain applications continues to grow, industry participants are likely to face heightened regulatory scrutiny, even as the regulatory landscape shifts and evolves with the technology. 
Blockchain is poised to become the most exciting invention after the Internet; while the latter connects the world to enable new business models based on online business processes, the former will help resolve the trust issue more efficiently via network computing. (Zhao, Fan, \& J. , 2016)

A Blockchain is a distributed append-only store of transactions distributed across computational nodes and structured as a linked list of blocks, each containing a set of transactions (Weber, Lu, Tran, Deshmukh, Gorski, $\&$ Strazds, 2019). Blockchain was introduced as the technology behind Bitcoin (Nakamoto, 2008). In general, there are two types of Blockchain networks: Permissioned or private Blockchains. These networks are proprietary networks that specific individuals or entities use to conduct transactions (such as a group of banks processing financial transactions). Permissionless or public Blockchains. (Prusty, 2018) These are opensource networks that anyone can access and use. Unlike the bitcoin Blockchain and other public networks, permissioned Blockchain networks are typically developed by companies for their own private commercial use. We use in our approach a Hybrid Blockchain. In best defined as the Blockchain that attempts to use the best of both private and public Blockchain solutions. A Hybrid Blockchain will mean controlled access and freedom at the same time. In simple terms, some processes are kept private and others public (Peters \& Panayi, 2015).

We begin with an overview of our system. As illustrated in Figure 1, the three entities comprising our system are users, interested in checking a product certificate; services, the issuer of such certificate; and nodes, entities entrusted with maintaining the Blockchain and a distributed private key-value data store in return for incentives. Note that while users in the system normally remain (pseudo) anonymous, we could store service profiles on the Blockchain and verify their identity.

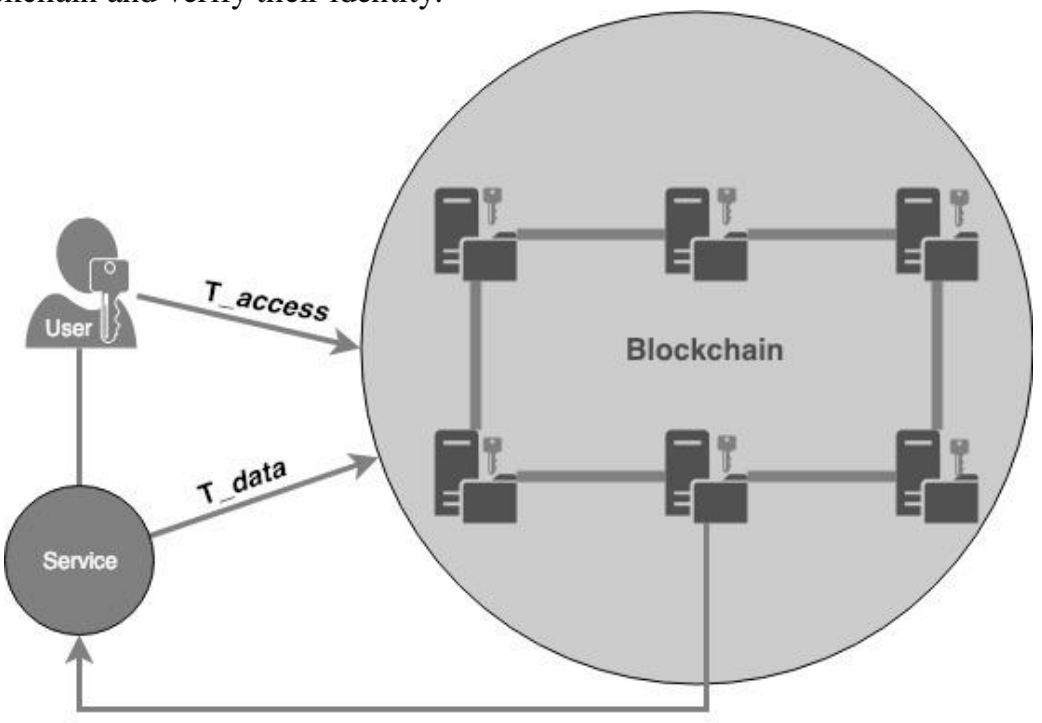

Figure 1. Certification Blockchain Service Overview (simplified)

The system itself is designed as follows. The Blockchain accepts two new types of Transactions: T_access, used for access certificate data; and $\mathbf{T} \_$data, for data storage and retrieval. These network operations could be easily integrated into a software development kit (SDK) that services can use in their development process. The Testing and inspection agencies or any product vendor could be drive such a service and publish his certification in the Blockchain.

For more understanding and before we compare our approach with the traditional one (centralized approach) we will describe our solution by using attributes:

Security The data used by this approach are secured by a network of distributed computers (nodes) around the world. Each one of these computers commits its computational power to make these databases secure and immutable.

Privacy Is any of my user data publicly visible? No, we use cryptography algorithms to register just a unique identifier of the personal data and we embed such identifier in the Blockchains. There is no way to reconstruct the content from any identifier because we use one-way cryptographic functions (hashes).

Transparency The approached Blockchain are fully distributed and transparent: anyone can access them from anywhere in the world. While the content is not stored in the Blockchains, the proofs of the validity, 
existence and ownership of such content can be independently verified by anyone by using the public keys, whether it be an end consumer, customs, a digital forensic or an authority.

Immutability an immutable record is an unchangeable record whose state cannot be modified after it is created. This in turn increases confidence in the integrity of the data and reduces the potential for fraud.

Global Authentication systems could be independently verified from anywhere in the world, even if the data is spread internationally among many organizations.

Validity with this approach consumer, authorities, industries and consumer-central have the opportunity to check the validity of certifications any time. The validity and other certificate information like date of issue, owner and date expired could not be manipulated. That is one of the most important features of decentralization.

To continue understanding this approach, the difference to the conventional way to access data needs to be demonstrated.

\section{COMPARISON BETWEEN CENTRALIZED-BASED AND DECENTRALIZED-BASED APPROACH}

To start with, we provide a short description about the types and capabilities of modern databases. Depending on the nature of the data one is storing, there are five genres of databases (Redmond \& Wilson, 2012).:

- Relational databases, such as SQL and variants, which are based on set theory and implemented as two-dimensional tables;

- Key-value stores, which store pairs of keys and values for fast retrieval;

- Columnar databases, which store data in columns, and can have more efficient representations of sparse tables compared to relational databases;

- Document databases;

- Graph databases, which model data as nodes and relationships.

There are centralized or distributed databases in our research we will focus on the latter because it is closer to the Blockchain concept. One of the objective of a distributed database is to partition larger information retrieval and processing problems into smaller ones, in order to be able to solve them more efficiently. In such databases, a user does not, as a general rule, need to be aware of the database network topology or the distribution of data across the different nodes. It should also be noted that in a distributed database, the connected nodes need not be homogeneous, in terms of the data that they store (Elmasri \& Navathe , 2015). Distributed database has several advantages (Elmasri \& Navathe, 2015, S. 882):

- Better reliability and availability, where localized faults do not make the system unavailable;

- Improved performance / throughput;

- Easier expansion.

One of the challenges in a distributed database environment is the issue of how modifications to the databases are propagated to the various nodes that should hold that data. The traditional approach is a 'master-slave' relationship, where updates to a master database are then propagated to the various slaves. However, this means that the master database can become a bottleneck for performance.

A Blockchain could be seen as a new type of distributed database which can help prevent such conflicts. In the same way that the virtual currency based on smart contracts will reject a transaction where the balance to be transferred has already been 'spent', a Blockchain can extend the operation of distributed databases by rejecting transactions which, e.g. delete a row that has already been deleted by a previous transaction (where a modification is a deletion, followed by the creation of a new row) (Gideon, 2017).

A second main difference between Blockchains and distributed databases lies in the ability to create self-enforcing contracts that will modify the Blockchain's data. Many permissioned Blockchains have a built-in virtual machine, such that one can execute pieces of computer code on the network. If this virtual machine is Turing-complete, this means that the machine can potentially solve a very large set of problems, which is very useful for executing more complex transactions on the network, possibly conditional on the state of certain off chain variables. (Gideon, 2017)

We can clearly see that the design is what makes a traditional database and Blockchain different. In this research we will shortly summarize the different in some points (Tabora, 2018):

- A database can be modified, managed and controlled by a single user called an administrator. Blockchain does not needs an administrator to validate a block of transactions. Instead of administrator are miners that perform this verification by solving cryptographic puzzles based on a difficulty level proportional to the total network hashing power available. 
- A database is also recursive, meaning that you can go back to repeat a task on a particular record and modify or delete it. Blockchain transactions are non-recursive, meaning they cannot be repeated once validated in a block. A Blockchain is highly fault tolerant since if one or more nodes are down, there will always be other nodes available that will run the Blockchain.

- A database is implemented in a client/server architecture. Blockchain uses a P2P network architecture. In the following section we build a proof of concept to check the feasibility of the researched approach.

\section{A PROOF OF CONCEPT FOR A BLOCKCHAIN-BASED APPROACH}

To demonstrate our approach, we design a certification architecture based on Bitcoin Blockchain. In details: a certificate issuer signs a well-structured certificate and stores its hash within a Blockchain transaction. A transaction output is assigned to the recipient. The next Figures describes how such services in the first detail degree works.

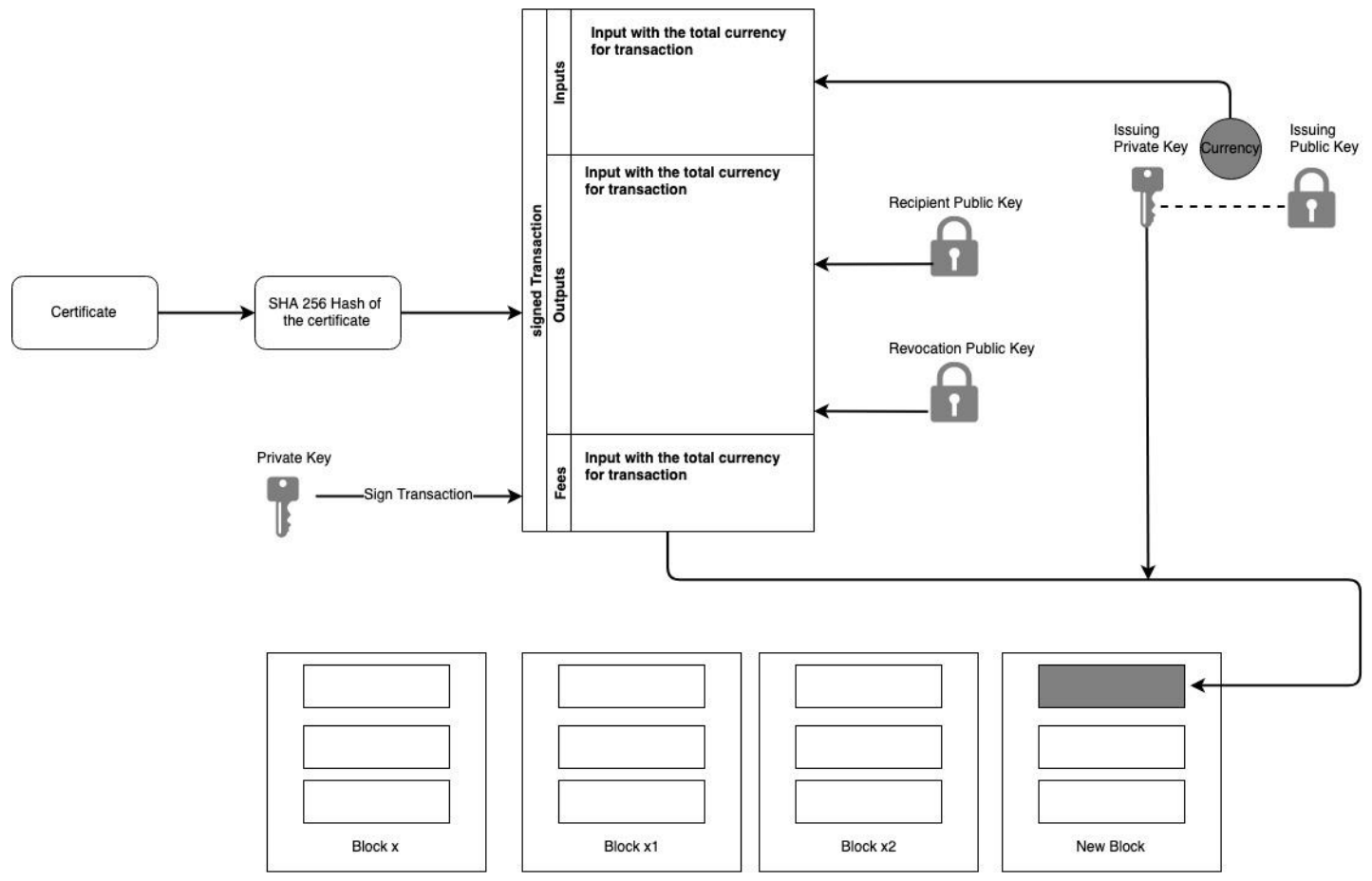

Figure 2. Overview of our high level certification Blockchain architecture

The following three repositories make up a certificates architecture:

- Certificate-Schema,

- Certificate-Issuer (certification body),

- $\quad$ Certificate-Viewer.

Certificate-schema describes the data standard for certificates. A certificate is essentially a JSON file with the necessary fields needed for our Certificate-issuer code to place it on the Blockchain.

Certificate-issuer ( $\mathbf{T}$ _data) takes a JSON certificate, creates a hash of the certificate, and issues a certificate by broadcasting a transaction from the issuing institution's address to a recipient's address with the hash embedded within output (valid or invalid) field.

Certificate-viewer ( $\mathbf{T}$ _access) is used to display and verify digital certificates after they have been issued. The viewer code also provides the ability for users to request certificates.

Why we implement the proof of concept in the Bitcoin Blockchain and not Ethereum-based Blockchain?

The answer that we know that Ethereum is better applicable for our idea which given more transparency and use of smart contract by using blockchain stored applications for contract negotiation and facilitation but 
Bitcoin has been the most tested and reliable blockchain to date; in addition, the relatively robust self-interest of miners, and the financial investment made into Bitcoin (Vukolić, 2016).

Our solution is not locked to one particular blockchain. it would be easy to also start publishing credentials to other blockchains, but for most of what we want to do, the functionality of the Bitcoin blockchain continues to be sufficient. What we did not investigate in thi spaper amongst other things what exactly does the smart contract use look like, but we believe such approaches can be more widely adopted to reduce transaction cost and expand functionality, as well as the topic of certificate revocation.

In the following section we discuss shortly the challenges of our approach like data integrity, confidentiality, privacy, the willingness to participate and sharing information aspects and comment on our Blockchain attributes mentioned in 3 with regard to them.

\section{CHALlengeS}

The challenges of decentralizing of transparency by using Blockchain to reduce counterfeiting are on the one hand data integrity, confidentiality on the Blockchain and on the other hand privacy and the willingness to participate and sharing information.

Companies and organizations use the data they collect to personalize services, optimize the corporate decision making process, predict future trends and more. If one is to consider how to incorporate such records onto a Blockchain there are fundamental issues of privacy to be considered.

Confidentiality involves the protection of data from unauthorized disclosure, either by direct retrieval or by indirect logical inference. Confidentiality considerations can also involve the possibility that information may be disclosed by legitimate users acting as an information channel, passing secret information to unauthorized users. Within a Blockchain, the choice of a permissioned or permissionless structure will define whether data will be made available to the public, or only within an organization. Permissionless Blockchains also enable carrying out transactions without the disclosure of private information. Because the operation of these Blockchains rests on public key cryptography, securing users' private keys is critical, and indeed, this is one of the main source of operational risks in this area (Peters G. C., 2016).

Maintaining the integrity of data entails its protection from invalid modification, insertion or deletion, thereby preserving the accuracy, consistency and validity of data over its life cycle. Ensuring this integrity is important for the recoverability and searchability, as well as the traceability and connectivity of certificate data records. This process usually requires a set of constraints or rules that define the correct states of the data set, and maintain correctness under operation. The subject of data integrity should be comprehensively investigated, maybe could some projects such as Enigma in MIT, see (Zyskind, Nathan, \& Pentland, 2015) support us in this.

Many of the most interesting challenges we encountered were not technical in nature like the willingness to participate and sharing information from testing and inspections agencies (certification bodies), but they cannot easily be separated from the technology because small design decisions can fundamentally shape behavior. That is why we have to do small experimental steps, tested the system with actual users and with one or two certification bodies, and continue to make changes based on what we are learning. The Blockchain is a relatively new technology and its complexity and immutability make it even more important to carefully consider the long-term effects of design decisions. After a short flight over the challenges we headline a brief summary and an outlook on our work.

\section{CONCLUSION AND FUTURE WORKS}

This paper presents a platform architecture to decentralizing products certificates using Blockchain Technology. We evaluate the solution by using a Bitcoin blockchain as a proof of concept We focused on feasibility in this paper. The following conclusions can be withdrawn from this new appoarch: we need to research more if a 'fully-open' blockchain implementations can reach the real goals and promise of blockchain in produkt certification services and counterfeiting of products. The adoption of blockchaine-based certificates has been held back by the ease with which they may be forged. The blockchain provides a way for certifications bodies to issue immutable digital certificates which are valid in perpetuity, since their authenticity can be 
verified against the blockchain. These advantages over database central systems significantly increase the value proposition of digital certificates, and will likely push digital certification into the mainstream.

In future work, we plan to explore and research more about using of smart contract and Ethereum-based Blockchain and like all prior disruptive technologies there will be beneficial and detrimental aspects of Blockchain technologies that will need to be carefully considered prior to development and commercialization of the approach presented in this paper.

However, we believe that with the onset of internet of certification by decentralizing of transparency, the Blockchain revolution will play an integral part in this brave new world.

\section{REFERENCES}

Directorate-General for Justice and Consumers (European Commission). (2018). Results of the EU rapid alert system for dangerous non-food products. European Commission, Directorate-General for Justice and Consumers. Brussels: European Commission.

Elmasri, R., \& Navathe, S. B. (2015). Fundamentals of Database Systems (Vol. 7). England: Pearson.

Ethereum Foundation (Stiftung Ethereum). (2018). Ethereum Project. (E. F. Ethereum), Producer, \& Ethereum Foundation (Stiftung Ethereum)) Retrieved 01 19, 2019, from Ethereum Project: https://www.ethereum.org/

Gideon, G. (2017). Blockchains vs Centalized databases. (Multichain, Producer, \& Multichain) Retrieved 01 19, 2019, from Blockchains vs Centalized databases: https://www.multichain.com/blog/2016/03/blockchains-vs-centralizeddatabases/

Nakamoto, S. (2008). Bitcoin: A Peer-to-Peer Electronic Cash System. https://bitcoin.org/bitcoin.pdf .

OECD/EUIPO. (2016). Trade in Counterfeit and Pirated Goods: Mapping the Economic Impact. Paris: OECD Publishing.

Peters, G. C. (2016). Opening discussion on banking sector risk exposures and vulnerabilities from Virtual currencies: An Operational Risk perspective. Journal of Banking Regulation .

Peters, G. W., \& Panayi, E. (2015). Understanding modern banking ledgers through blockchain technologies: Future of transaction processing and smart contracts on the internet of money. Computers and Society (cs.CY); Cryptography and Security (cs.CR), 5-6.

Prusty, N. (2018). Blockchain for Enterprise: : Build scalable blockchain applications with privacy, interoperability, and permissioned features (Vol. 1). Birgmingham, UK.

Redmond , E., \& Wilson, J. R. (2012). Seven Databases in Seven Weeks: A Guide to Modern Databases and the NoSQL Movement. North.

Remeur, C. (2017). Market surveillance and product safety. European Parliament. Bruessel: European Parliament.

Sirpa, P. (., Françoise, G. (., \& Bart, S. (. Member States should stop turning a blind eye to product safety. European Commission. The Parliament Magazine.

TÜV SÜD AG . (n.d.). Misuse of quality marks . Retrieved 01 12, 2019, from Misuse of quality marks : https://www.tuevsued.de/product-testing/_services/markets_and_competition/quality_and_test_marks/abuse_of_quality_marks

TÜV SÜD AG. (n.d.). Certificate Explorer. Retrieved 01 12, 2019, from Certificate Explorer: https://www.tuevsued.de/product-testing/certificates

Tabora, V. (2018, 08 04). Hacker Noon. (medium) Retrieved 01 19, 2019, from Hacker Noon: https://hackernoon.com/databases-and-blockchains-the-difference-is-in-their-purpose-and-design-56ba6335778b

The Netherlands Court of Audit. (2017). Products sold on the European market: unravelling the system of CE marking. The Netherlands Court of Audit. Netherlands: House of Representatives.

Vukolić, M. (2016). The quest for scalable blockchain fabric: Proof-of-work vs. BFT replication. In Lecture Notes in Computer Science (including subseries Lecture Notes in Artificial Intelligence and Lecture Notes in Bioinformatics). 9591. Zurich: Springer Verlag.

Weber, I., Lu, Q., Tran, A. B., Deshmukh, A., Gorski, M., \& Strazds, M. (2019). A Platform Architecture for Multi-Tenant Blockchain-Based Systems. IEEE International Conference on Software Architecture (ICSA2019) , 10.

Zhao, J., Fan, S., \& J. , F. (2016). Overview of business innovations and research opportunities in blockchain and introduction to the special issue. Financial Innovation, 2 (1), 28.

Zyskind, G., Nathan, O., \& Pentland, A. (2015). Enigma: Decentralized Computation Platform with Guaranteed Privacy. Cryptography and Security. 